\title{
Assessing polarization effects for the Airborne imaging spectrometer APEX
}

\author{
U. Böttger ${ }^{1}$, J. Nieke $^{2}$, and D. Schläpfer ${ }^{2}$ \\ ${ }^{1}$ DLR, Institut für Planetenforschung, Berlin, Germany \\ ${ }^{2}$ Remote Sensing Laboratories University of Zurich, Switzerland
}

\begin{abstract}
In the scope of hyperspectral airborne imaging spectrometer (APEX) design activities, the acceptable sensitivity of linear polarization of the spectrometer is analyzed by assessing the amount of polarization of reflected light in the atmosphere-surface system. A large number of calculations is performed for a wide variaty of viewing geometries to study the influences of aerosol models, natural surfaces and flight altitudes over the spectral range from the near-UV to the short-wave infrared (SWIR).

Thereinafter the design of the imaging spectrometer is outlined accounting for these requirements and a method of partially correcting the instrument polarization sensitivity is briefly introduced. APEX design and post-processing capabilities will enable to reduce the influence of polarization sensitivity of at-sensor radiance and its higher-level products generated for most of the observation conditions.
\end{abstract}

\section{Introduction}

In the remote sensing community, it is assumed implicitly that satellite or airborne sensors are able to measure accurately the incoming at-sensor radiance assuming a perfectly calibrated system. However, the at-sensor radiance reflected from the atmosphere-surface system can be strongly polarized (Böttger et al., 2005) and sensors not generally designed for being insensitive to the polarization state of the radiance tend to generate a biased measurement. The sensitivity of the optical sensor to the incoming polarized radiance is called in the following "self-polarization effect".

Since polarization of light passing an atmosphere-surface system may affect accurate measurements of earth observing optical instruments, various approaches exist to account for this effect in the retrieved data product: (1) One is to measure the polarization of the incoming radiation. This allows the

Correspondence to: U. Böttger (Ute.Boettger@dlr.de) use of additional information for better retrieval of parameters of the observed system (Herman et al., 1997; Leroy et al., 1997; Deuze et al., 2001a,b; Goloub et al., 1999). (2) Another possibility is to reduce the self-polarization by a polarization measurement device, e.g. PMD for GOME (GOME, 1995) or the PMU for GOME-2 (GOME2, 2005).

In this paper we discuss a third method for the Airborne Prism EXperiment (APEX), a hyperspectral imaging spectrometer, which is currently being built in a joint Swiss/Belgium project funded through ESA (Itten et al., 1997; Nieke et al., 2004). The optical design of the APEX instrument does not foresee additional measurements of the polarization state of the incoming radiance and very efficient scramblers would lead to a degraded point spread function, i.e. a loss of spatial resolution. This is why the APEX imaging spectrometer will make use of a removable scrambler system and post-processing routines using the spectrometer inherent information in the transitions zone between two spectral channels.

In the first part of this paper, the project background, the optical design and the key requirements of the APEX instrument are summarized. In the second part, we review the degree of polarization expected at-sensor flight levels for a large number of viewing angles, aerosol models, natural surfaces and flight altitudes and over the spectral range from the near-UV until the short-wave infrared (SWIR). The calculations are based on radiative transfer calculations performed with the radiative transfer code STORM (STOkes vector Radiative transfer Model) (Böttger et al., 2005). As result we retrieve the polarization variability of the radiation due to effects of atmosphere and surface, which allowes to define the maximum acceptable instrumental polarization sensitivity. In the third part, we propose an imaging spectrometer design solution to reduce the self-polarization of APEX to acceptable values. A removable scrambler system is implemented to enable high spatial resolution measurements. Moreover, post-processing allows the assessment of the po-

Published by Copernicus GmbH on behalf of the URSI Landesausschuss in der Bundesrepublik Deutschland e.V. 
Table 1. APEX instrument - Key Requirements.

\begin{tabular}{|c|c|}
\hline Parameter & Value \\
\hline $\begin{array}{l}\text { Field of View (FOV) - } \\
\text { pushbroom technique }\end{array}$ & $\begin{array}{l}\text { Swath: } \pm 14 \text { deg } \\
\text { with } 1000 \text { across-track pixel }\end{array}$ \\
\hline $\begin{array}{l}\text { Instantaneous Field of View } \\
\text { (IFOV) }\end{array}$ & $0.48 \mathrm{mrad}$ \\
\hline Flight altitude range & $3500-10000$ m.a.s.l. \\
\hline Standard aircraft interface & $\begin{array}{l}\text { for Dornier Do- } 228 \\
\text { on stabilizing platform PAV-30 }\end{array}$ \\
\hline Spectral range & $\begin{array}{l}\text { VNIR: } 380-1000 \mathrm{~nm} \\
\text { SWIR: } 940-2500 \mathrm{~nm}\end{array}$ \\
\hline Spectral bands & $\begin{array}{l}\text { VNIR: } 312 \text { (prior binning) } \\
\text { SWIR: } 199\end{array}$ \\
\hline Spectral sampling interval & $\begin{array}{l}380-1050 \mathrm{~nm}: ~ i 5 \mathrm{~nm} \\
1050-2500 \mathrm{~nm}: i 10 \mathrm{~nm}\end{array}$ \\
\hline Spectral resolution & $\leq 1.75$ Sampling interval \\
\hline
\end{tabular}

larization state and reduction of instrumental error due to self-polarization.

\section{The APEX instrument}

APEX (Airborne Prism EXperiment) is a Swiss-Belgian project (Nieke et al., 2004) of the European Space Agency ESA that contributes to the preparation, calibration, validation, simulation, and application development of future remote sensing missions in space, as well as to the understanding of land and coastal processes and interactions at a local and regional (or national) scale.

Since APEX samples hundreds of narrow contiguous spectral bands throughout the visible and short wave infrared electromagnetic spectrum, it provides relevant input to numerous applications, such as vegetation analysis and forestry, agriculture, snow and ice applications, coastal zone management, aquatic environments, soil monitoring, atmospheric and geological applications, security and emergency response, policy and space.

The instrumentation comprises a hyperspectral imager compatible to various airborne platforms (such as Do-228, the High Altitude and LOng Range Research Aircraft HALO (HALO, 2005)), a fixed installed calibration home base (Suhr et al., 2005) and a complete facility for data processing and archiving (Schläpfer et al., 2003; Kaiser et al., 2003; Brazile et al., 2005). The pushbroom-type instrument accommodates two spectrometer channels and covers a spectral range from $0.38 \mu \mathrm{m}$ up to $2.5 \mu \mathrm{m}$ (Table 1 ). The spatial/spectral resolution amounts to 1000 spatial samples at 28-degree field of view with more than 300 spectral bands. The overall instru- ment design and its built-in characterization unit will allow stable performance under various flight conditions.

According to the optical design of the instrument the scene is imaged by the ground imager via a path-folding mirror onto the spectrometer slit with $40 \mu \mathrm{m}$ slit width. Filters will be available for radiance level adaptation and calibration purposes. A collimator optic directs the light to the prisms. For compensation of the distortion introduced by the prisms, a small spherical mirror is located in the collimator image field. For separating the SWIR and VNIR channel, the first dispersion prism carries on its second surface a dichroic beamsplitter coating, with high reflectivity in the VNIR region. In order to minimize the polarization sensitivity of the instrument, a scrambler (or depolarizer (Hanle, 1931)) can be attached in front to randomize the polarization of the incoming light. Details on the design and the achieving polarization sensitivities are described in Sect. 4.

\section{Polarization of light in an atmosphere-surface system}

Radiation in the visible and near infrared spectral region is considerably polarized (Coulson, 1988). The Stokes parameters $(I, Q, U, V)$ are used to describe the full state of radiation (Chandrasekhar, 1950; Liou, 1980): $I$ is the intensity. $Q, U$ describe the part of linear polarized light and the plane of polarization. $V$ discriminates between linear, elliptic or circular polarization. The degree of polarization is defined as

$P_{\mathrm{deg}}=\frac{\sqrt{Q^{2}+U^{2}+V^{2}}}{I}$

The plane of polarization $\chi$ is defined as

$\tan 2 \chi=\frac{U}{Q}$

In the case of APEX only radiance measurements are of interest and the degree of polarization is the parameter that characterizes the error due to instrumental polarization sensitivity adequately. It describes the percentage of radiation that is affected by polarization and thus can contribute to the instrumental error. The plane of polarization is necessary for correction purposes as explained later.

Polarization in the visible and near infrared spectral range is caused by interaction of the incoming unpolarized solar irradiance with the atmosphere and the surface. The important processes causing polarization of light in nature are scattering at gas, aerosol and cloud particles in the atmosphere and reflection at soil, sand, vegetation, water and other surface types. Only linear polarization is assumed for the system of atmosphere and surface. Processes producing considerable circular polarization can be neglected in the observed system (Coulson, 1988). The strongest features of the degree of polariaztion can be found in the principal plane (the viewing angle plane formed by the sun incident and zenith/nadir direction). 
Table 2. Medium of the degree of polarization in steps of $5 \%$ for the IFOV of Apex for aerosol loaded atmospheres (see text) for different surfaces (albedo 0.0, 0.05 and 0.2 and a water ruffled surface) at a flight altitude of $\mathrm{MOA}=3000 \mathrm{~m}$ and BOA: bottom of atmosphere and for the absorption free or weakly absorbing wavelengths $(\lambda=380 \mathrm{~nm}, 443 \mathrm{~nm}, 670 \mathrm{~nm}, 865 \mathrm{~nm}, 1150 \mathrm{~nm}, 2140 \mathrm{~nm})$. The sun zenith is less than $40^{\circ}$.

\begin{tabular}{llrrrrrr}
\hline surf & Height & $\begin{array}{r}380 \\
\mathrm{~nm}\end{array}$ & $\begin{array}{r}443 \\
\mathrm{~nm}\end{array}$ & $\begin{array}{r}670 \\
\mathrm{~nm}\end{array}$ & $\begin{array}{r}865 \\
\mathrm{~nm}\end{array}$ & $\begin{array}{r}1150 \\
\mathrm{~nm}\end{array}$ & $\begin{array}{r}2140 \\
\mathrm{~nm}\end{array}$ \\
\hline 0.00 & MOA & $<5$ & 5 & 5 & 5 & 5 & 10 \\
0.05 & MOA & $<5$ & $<5$ & $<5$ & $<5$ & $<5$ & $<5$ \\
0.20 & MOA & $<5$ & $<5$ & $<5$ & $<5$ & $<5$ & $<5$ \\
\hline \multirow{2}{*}{ water } & MOA & 5 & 5 & 5 & 5 & 10 & 10 \\
& BOA & 5 & 5 & 5 & 5 & 10 & 10 \\
\hline
\end{tabular}

Table 3. Medium of the degree of polarization in steps of 5\% for the IFOV of Apex for aerosol loaded atmospheres (see text) for different surfaces (albedo 0.0, 0.05 and 0.2 and a water ruffled surface) at a flight altitude of $\mathrm{MOA}=3000 \mathrm{~m}$ and $\mathrm{BOA}$ : bottom of atmosphere and for the absorption free or weakly absorbing wavelengths $(\lambda=380 \mathrm{~nm}, 443 \mathrm{~nm}, 670 \mathrm{~nm}, 865 \mathrm{~nm}, 1150 \mathrm{~nm}, 2140 \mathrm{~nm})$. The sun zenith is larger than $40^{\circ}$.

\begin{tabular}{rlrrrrrr}
\hline surf & Height & $\begin{array}{r}380 \\
\mathrm{~nm}\end{array}$ & $\begin{array}{r}443 \\
\mathrm{~nm}\end{array}$ & $\begin{array}{r}670 \\
\mathrm{~nm}\end{array}$ & $\begin{array}{r}865 \\
\mathrm{~nm}\end{array}$ & $\begin{array}{r}1150 \\
\mathrm{~nm}\end{array}$ & $\begin{array}{r}2140 \\
\mathrm{~nm}\end{array}$ \\
\hline 0.00 & MOA & 15 & 20 & 20 & 20 & 25 & 35 \\
0.05 & MOA & 10 & 10 & 10 & 10 & 10 & 5 \\
0.20 & MOA & 5 & 5 & 5 & 5 & 5 & $<5$ \\
\hline \multirow{2}{*}{ water } & MOA & 10 & 15 & 20 & 20 & 25 & 30 \\
& BOA & 10 & 15 & 15 & 15 & 15 & 20 \\
\hline
\end{tabular}

The polarization of radiation reaching the sensor results from polarizing and depolarizing effects of atmosphere and surface. Herman et al. (1997) showed the influence of the different (molecular and aerosol) contributions of atmospheric polarized radiance to the polarized part of the ground reflectance. It is important to consider atmospheric polarization even if only the surface radiation is of interest. In the Tables 2 to 4 the maxima and medium values of the degree of polarization are shown, that are expected within the field of view (FOV) of APEX for different atmopshere - surface systems and for two sets of sun incident angles (less and larger than $40^{\circ}$ ). These values are derived from radiative transfer calculations of the Stokes parameters with the radiative transfer code STORM (Böttger et al., 2005). STORM is used
Table 4. Maximum of the degree of polarization in steps of 5\% for the IFOV of Apex for aerosol loaded atmospheres (see text) for different surfaces (albedo 0.0, 0.05 and 0.2 and a water ruffled surface) at different flight altitudes (TOA: top of atmosphere, MOA: $3000 \mathrm{~m}$, BOA: bottom of atmosphere) and for the absorption free or weakly absorbing wavelengths $(\lambda=380 \mathrm{~nm}, 443 \mathrm{~nm}$, $670 \mathrm{~nm}, 865 \mathrm{~nm}, 1150 \mathrm{~nm}, 2140 \mathrm{~nm}$ ).

\begin{tabular}{cccccccc}
\hline surf & Height & $\begin{array}{r}380 \\
\mathrm{~nm}\end{array}$ & $\begin{array}{r}443 \\
\mathrm{~nm}\end{array}$ & $\begin{array}{r}670 \\
\mathrm{~nm}\end{array}$ & $\begin{array}{r}865 \\
\mathrm{~nm}\end{array}$ & $\begin{array}{r}1150 \\
\mathrm{~nm}\end{array}$ & $\begin{array}{r}2140 \\
\mathrm{~nm}\end{array}$ \\
\hline \multirow{2}{*}{0.00} & TOA & 60 & 65 & 70 & 65 & 60 & 65 \\
& MOA & 45 & 55 & 60 & 55 & 55 & 70 \\
\hline \multirow{2}{*}{0.05} & TOA & 55 & 55 & 35 & 25 & 25 & 20 \\
& MOA & 30 & 30 & 20 & 20 & 25 & 20 \\
\hline & TOA & 45 & 35 & 20 & 15 & 10 & 5 \\
& MOA & 15 & 15 & 10 & 10 & 10 & 5 \\
\hline \multirow{2}{*}{ water } & TOA & 55 & 65 & 65 & 65 & 65 & 70 \\
& MOA & 40 & 50 & 60 & 60 & 60 & 70 \\
& BOA & 40 & 45 & 50 & 55 & 60 & 70 \\
\hline
\end{tabular}

to perform a large number of calculations covering the full range of remote sensing. These values will be underpinned by statements from literature (see this volume, Böttger et al.). The calculations are performed for nine wavelengths $(380 \mathrm{~nm}, 443 \mathrm{~nm}, 670 \mathrm{~nm}, 865 \mathrm{~nm}, 929 \mathrm{~nm}, 937 \mathrm{~nm}, 943 \mathrm{~nm}$, $1250 \mathrm{~nm}$, and $2140 \mathrm{~nm}$ ). Except the wavelengths of $929 \mathrm{~nm}$, $937 \mathrm{~nm}, 943 \mathrm{~nm}$ all other wavelengths are chosen as non or weakly absorbing.

The scattering characteristics of aerosols are determined by Mie theory. Non-spherical particles like cirrus ice clouds are not considered. The atmospheric layering corresponds to the proposal of WCP (1986). The surface is described by a Lambertian reflector of albedo of $0 \%, 5 \%$ and $20 \%$ and by a wind ruffled water surface as an extreme anisotropic reflection case. Five boundary layer aerosol types (maritime 70\% and $99 \%$ humidity, continental, urban $80 \%$ humidity and a mixture of continental and maritime $99 \%$ humidity) are considered. The aerosol optical thickness at 550nm ranges between 0.05 and 1.2 with the focus on lower optical thickness. The sensor altitude is fixed at $0 \mathrm{~km}, 3 \mathrm{~km}, 5 \mathrm{~km}, 7 \mathrm{~km}$ and at top of atmosphere, representing typical situations for ground based, airborne, and space borne spectro-radiometers. 


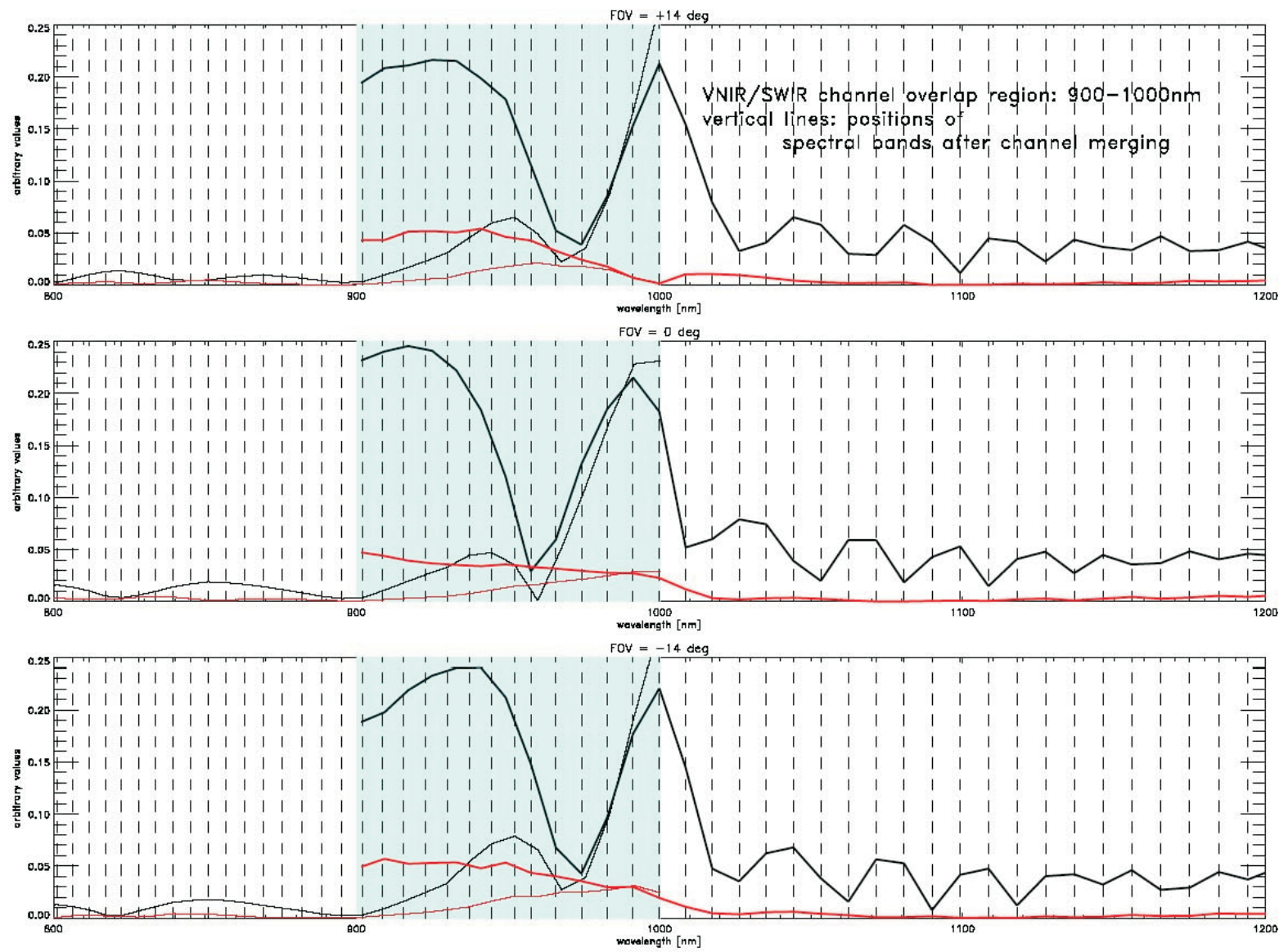

Fig. 1. Polarization sensitivity of the APEX imaging spectrometer in the transition region range from $900 \mathrm{~nm}$ to $1000 \mathrm{~nm}$ for the sensor viewing angles $F O V=+14^{\circ}, 0^{\circ},-14^{\circ}$ and for two scrambles (scrambler 1: black line, scrambler 2: red line). The location of the center wavelength of the APEX spectral bands in plotted as vertical dashed line.

\section{Polarization issues in APEX design}

The above outlined calculations lead to a polarization requirement for the optical system design of an airborne imaging spectrometer, such as APEX. For a baseline design standard setting with a flight altitude of $3 \mathrm{~km}$ and a total FOV of 28 degrees are chosen.

The numbers given in the Tables 2 to 4 lead to an expected at sensor polarization of $20-30 \%$ for the Lambertian surface case at typical dark objects of $5 \%$ reflectance. From these numbers it can be concluded that the degree of at sensor polarization is not above $30 \%$ over land. If now a $1 \%$ uncertainty shall be allowed in the final measurements due to polarization, the sensor shall be designed such that its polarization sensitivity in the visible and near infrared is below $3 \%$. In the shortwave infrared spectral range, the surface reflectance is in average significantly higher than for shorter wavelengths which allows an increase of the allowed sensitivity to $5 \%$.

In the APEX design various solutions were included to reduce the self-polarization sensitivity implementing hardware and software related tools, i.e. (1) a removable scrambler system in front of the entrance optics of the APEX instrument and (2) applying post processing algorithms using the information in the transition zone, i.e. an overlapping spectral region between the two detectors.

\subsection{APEX scrambler system}

A removable polarization scrambler system is situated in the light path of APEX. The scrambler system allows to mount two different depolarizers (or scrambler) in front of the APEX entrance optics with the goal to optimize the system for special scientific user needs. Both scramblers consist of three optically contacted wedges, one of which is birefringent and affects the polarization state differently at different locations. The scrambler transforms one polarization state into a continuum of polarization states. The other wedges compensates for the prismatic deviation. Whereas the divergence of the incoming light beams polarized at right angles to each other is about 8 arc minutes for scrambler 1 , the divergence is 24 arc minutes for scrambler 2. For the 
APEX optics, the requirement on the polarization sensitivity can be reached with a scrambler having 24 arc minutes, however with the disadvantage of decreasing the geometric resolution and uniformity of the instrument. The application of scramblers in an imaging spectrometer design leads always to the tradeoff between improved polarization sensitivity vs. worsen geometric performance, i.e. misregistration of the spectro-spatial information. For the selected scramblers, the spectral misregistration is not influenced since the spectral dispersion is generated behind the slit in a duplex prism. For the spatial misregistration, the 8 arc minutes scrambler-1 causes negligible influence on the image quality.

However, the utilization of scambler- 2 has an effect on the spatial misregistration. The scrambler can cause a change in spatial misregistration in the across track direction up to almost 0.1 pixel. As for the along track direction, the error is quite large and can become almost 0.4 pixels. Whereas the spatial misregistration error in the across track direction is relatively insensitive to polarization direction, the along track error depends on wavelength and viewing geometry.

\subsection{Post processing}

APEX is designed to be a well-calibrated optical instrument over the whole wavelength range. As such, the transition zone between the two detectors will be sampled by both detectors accurately enough to make use of the special behavior of the beam splitter. Non-polarized radiance will be measured at the same levels for both calibrated detectors. Any difference between corresponding spectral bands can then be attributed to polarization in the signal. If polarization is significant it will be visible between $930-960 \mathrm{~nm}$ (Fig. 1) and will allow a quantification of the degree of polarization at these wavelengths. A transfer of this self-consistent measurement to the whole SWIR wavelength range is feasible by thorough models and through polarization dependent laboratory measurements of the instrument response. Based on the radiative transfer simulations mentioned in Sect. 3, a spectral correlation analysis for the degree of polarization (Fig. 2) implies the possibility of polarization correction as part of post processing for non or weakly absorbing wavelength for sun zenith angles larger than $40^{\circ}$, where the degree of polarization has considerable values. In Fig. 2, the spectral correlation coefficient between $943 \mathrm{~nm}$ and $1250 \mathrm{~nm}$ and between $1250 \mathrm{~nm}$ and $2140 \mathrm{~nm}$ for the degree of polarization and the plane of polarization is shown. The vector used for determination of correlation coefficient contains the degree of polarization for four surface types and 16 atmospheric conditions. Every case on the abscissa depends on a specific sun zenith angle (larger $40^{\circ}$ ), viewing angle in the IFOV of APEX and height. The degree of polarization shows a very high spectral correlation in the SWIR for more or less absorption free wavelengths. Additionally, the plane of polarization is relatively independent of wavelength and is mainly determined by viewing geometry. Based on this fact, a post processing
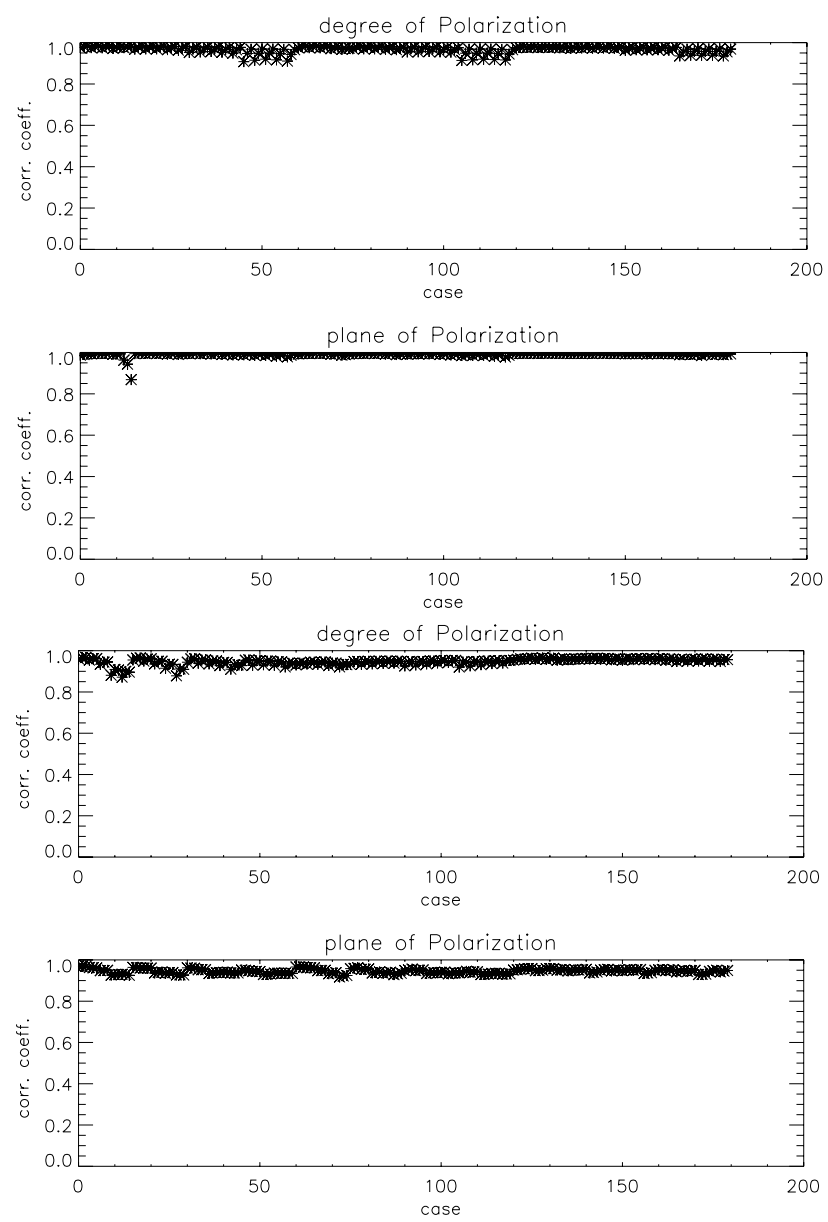

Fig. 2. Spectral correlation between $943 \mathrm{~nm}$ and $1250 \mathrm{~nm}$ (upper plots) and between $1250 \mathrm{~nm}$ and $2140 \mathrm{~nm}$ (lower plots) for the degree of polarization and the plane of polarization. The vector used for determination of correlation coefficient contains the degree of polarization for four surface types and 15 atmospheric conditions. The cases on the abscissa depend on sun zenith angle (larger $40^{\circ}$ ), viewing angle in the IFOV of APEX and height.

procedure may be included in the APEX processing chain. The result of this post processing will be a measurement corrected so as it is "normalized" to a predefined viewing geometry and thus the polarization errors due to the relative orientation of the instrument are eliminated.

A small example will illustrate the procedure: the degree of polarization of radiation at a wavelength of interest reaching the sensor may be $P_{a}=0.2$ (or $20 \%$ ). This value is derived based on the spectral correlation of the degree of polarization between the overlap region of the two scramblers and the wavelength of interest in the SWIR. The self-polarization of the instrument at the considered wavelength derived from laboratory measurements may be $P_{i}=0.1$ (or 10\%). Thus maximum $P_{a} * P_{i}=0.02$ (or $2 \%$ ) of the total signal need to be corrected for self-polarization in our example. The predefined viewing geometry is described by the angle $\chi_{i p}$ be- 
tween the principal plane and the instrumental plane, taken as $0^{\circ}$. If the instrumental plane of self-polarization is $\chi_{i p}=90^{\circ}$ to the principal plane, then the measurement has to be increased by $P_{a} * P_{i} * \sin \left(90^{\circ}\right)=0.02$ (or $2 \%$ ). For any other angle between the instrumental plane and the principal plane the value of 0.02 (or $2 \%$ ) must be multiplied by $\sin \left(\chi_{i p}\right)$ and added to the measurement value. With this approach all measurements are comparable, because they are "normalized" to a predefined geometry and the error due to self-polarization is minimized. This is valid only for absorption free or weakly absorbing wavelengths. If absorption takes place, especially in the wings of absorption bands, the spectral correlation disappears. That means that the degree of polarization shows an unpredictable high spectral variability. As the scrambler produces a high spectral self-polarization variability as well, it is proposed in this case to perform measurements without the scrambler to avoid artificial spectral features.

The postprocessing procedure for non and weakly absorbing wavelengths has the potential to lead to a further improvement of the sensor polarization sensitivity. The such measured degree of polarization may even be used to derive additional products from the APEX system, which describe environmental parameters such as aerosol properties based on their polarization state.

\section{Conclusions}

By means of simulations we demonstrate that the atmosphere-surface system can be strongly polarized and sensors not generally designed for being insensitive to the polarization state of the radiance and tend to generate significant errors in the at-sensor radiance products. It is concluded, that the bias in the measurements due to polarization should be as small as possible, resulting in a polarization sensitivity design specification of the APEX of less than $3 \%$ in the VNIR and less than 5\% in the SWIR. Accounting for this specification and realizing also a high spatial resolution of the imaging spectrometer, the APEX project selected an optical design trade-off making use of a removable scrambler system and post-processing routines benefiting from the spectrometer's inherent information in the transitions zone between two spectral channels. Consequently, the APEX sensor will deliver high spectral and spatial resolution data over a wide spectral range accounting also for the instrumental polarization sensitivity in a way that the measured atsensor radiance are biased within acceptable limits for most of the science applications.

\section{References}

Böttger, U., Preusker, R., and Nieke, J.: Radiative transfer model STORM for full Stokes vector calculations for a plane parallel atmosphere-surface-system, submitted to SPIE, Remote Sensing of Clouds and the Atmosphere X conference, 2005.
Brazile, J., Kaiser, J., Schläpfer, D., Nieke, J., Schaepman, M., and Itten, K.: Parallization of APEX airborne imaging spectrometer product generation, submitted to e-proceedings EARSeL, 2005.

Chandrasekhar, S.: Radiative Transfer, Oxford University Press, Oxford, 1950.

Coulson, K.: Polarization and Intensity of Light in the Atmosphere, A. Deepak Publ., Hampton, Virginia, USA, 1988.

Deuze, J. L., Breon, F., Devaux, C., Goloub, P., Herman, M., Lafrance, B., Maignan, F., Marchand, A., Nadal, F., and Tanre, D.: Remote sensing of aerosols over land surfaces from POLDER-ADEOS-1 polarized measurements, J. Geophys. Res., 106, 4913-4926, $2001 \mathrm{a}$.

Deuze, J. L., Goloub, P., Herman, M., Marchand, A., Perry, G., Susana, S., and Tanre, D.: Estimate of the aerosol properties over the ocean with POLDER, J. Geophys. Res., 106, 4913-4926, $2001 b$.

Goloub, P., Tanre, D., Deuze, J., Herman, M., Marchand, A., and Breon, F.: Polarized Reflectance of Bare Soils and Vegetation: Measurements and Models, IEEE Transactions on Geoscience and Remote Sensing, 37, 1586-1596, 1999.

GOME: User Manual, Publication ESA SP-1182, ESA/ESTEC, 1995.

GOME2: http://www.sron.nl/www/code/eos/gome2/ gome2_PMDs.php/, 2005.

HALO: http://www.pa.op.dlr.de/halo, 2005.

Hanle, W.: Messung des Polarisationsgrades von Spektrallinien, Zeitschrift fr Instrumentenkunde, 51, 488, 1931.

Herman, M., Deuze, J. L., Devaux, C., Goloub, P., Breon, F. M., and Tanre, D.: Remote sensing of aerosols over land surfaces including polarization measurements and application to POLDER measurements, J. Geophys. Res., 102, 17 039-17 049, 1997.

Itten, K., Schaepman, M., Vos, L. D., Hermans, L., Schlaepfer, D., and Droz, F.: APEX - Airborne PRISM Experiment:A new concept for an airborne imaging spectrometer, in: 3rd Intl. Airborne Remote Sensing Conference and Exhibition, 1, 181-188, 1997.

Kaiser, J., Schläpfer, D., Brazile, J., Strobl, P., and Schaepman, M.: Assimilation of heterogeneous calibration measurements for the APEX spectrometer, 5234, 211-220, 2003.

Leroy, M., Deuze, J. L., Breon, F. M., Hautecoeur, O., Herman, M., Buriez, J., Tanre, D., Bouffies, S., Chazette, P., and Roujean, J.: Retrieval of atmospheric properties and land surface bidirectional reflectances over land from POLDER/ADEOS, J. Geophys. Res., 102, 17 023-17 037, 1997.

Liou, K. N.: An Introduction to Atmospheric Radiation, Academic Press, 1980.

Nieke, J., Itten, K., and APEX team: APEX: Current Status of the Airborne Dispersive Pushbroom Imaging Spectrometer, SPIE, 5542, 109-116, 2004.

Schläpfer, D., Kaiser, J., Brazile, J., Schaepman, M., and Itten, K.: Calibration concept for potential optical aberrations of the APEX pushbroom imaging spectrometer, SPIE, 5234, 221-231, 2003.

Suhr, B., Gege, P., Strobl, P., Kaiser, J., Nieke, J., Itten, K., and Ulbrich, G.: Calibration Home Base for the Airborne Imaging Spectrometer APEX, submitted to eproceedings EARSeL, 2005.

WCP: A preliminary cloudless standard atmosphere for radiation computation, Tech. rep., WCP-Report 112, WMO/TD-No.24, 1986. 